\title{
Characteristics of urgent palliative cancer care consultations encountered by radiation oncologists
}

\author{
Muhammed Mohsin Fareed, MD, ${ }^{\text {ab }}$ Alexander Spektor, MD, $\mathrm{PhD},{ }^{\mathrm{a}}$ Monica Krishnan, \\ MD, ${ }^{a}$ Lauren M Hertan, MD, ${ }^{c}$ Allison Taylor, MS, ${ }^{a}$ Andrea Kelly, MS, ${ }^{a}$ Mai Anh Huynh, \\ $\mathrm{MD}, \mathrm{PhD},{ }^{\mathrm{a}}$ and Tracy A Balboni, $\mathrm{MD}, \mathrm{MPH}^{\mathrm{ab}}$
}

${ }^{a}$ Department of Radiation Oncology, Brigham and Women's Hospital/Dana-Farber Cancer Institute; 'Department of Psychosocial Oncology and Palliative Care, Dana-Farber Cancer Institute; and 'Department of Radiation Oncology, Beth Israel Deaconess Medical Center, all in Boston, Massachusetts

\begin{abstract}
Background Palliative radiation therapy (PRT) is often used in patients with advanced cancers who require urgent consultation. Objective To report on the characteristics of urgent palliative issues encountered by radiation oncologists.

Methods Patterns of presentation in 162 consultations for urgent PRT at 3 centers were prospectively evaluated from May 19 to September 26, 2014. A survey of palliative care issues, including assessment of reasons for urgent consultation, disease presentation, characteristics, and sites of RT delivery, was completed by physicians and/or nurse practitioners. The response rate was $86 \%$, with 140 of 162 responses received.

Results The median age of the patients was 63 years, with $80 \% \geq 50$ years. $56 \%$ were men, and $44 \%$ were women. $57 \%$ had an Eastern Cooperative Oncology Group Performance Status of 0-1. Primary cancer diagnoses were lung (28\%), breast (13\%), prostate $(10 \%)$, melanoma $(10 \%)$, sarcoma $(7 \%)$, and others $(32 \%)$. The main reasons for PRT consult were pain $(57 \%)$, brain metastases $(29 \%)$, and cord compression (13\%). The most common presenting symptoms were pain (69\%), neurologic symptoms $(51 \%)$, and fatigue $(49 \%)$. Patients were seen throughout the trajectory of their care as follows: $63 \%$ at the time of an established metastasis, $19 \%$ at the time of their initial diagnosis continuing further cancer therapies, and $16 \%$ before hospice care without further anticancer therapy.

Limitations Single institution and descriptive

Conclusions PRT occurs across the spectrum of advanced cancer, from initial diagnosis to end of life, and is used in a range of urgent oncologic issues, mostly painful metastases, followed by brain metastases and cord compression. Radiation oncologists manage cancer-related symptoms such as pain, neurologic symptoms, and fatigue.
\end{abstract}

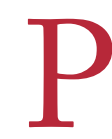
alliative radiation therapy (PRT) plays a major role in the management of incurable cancers. Study findings have demonstrated the efficacy of using PRT in treating tumor-related bone pain, ${ }^{1}$ brain metastases and related symptoms, ${ }^{2}$ thoracic disease-causing hemoptysis or obstruction, ${ }^{3}$ gastrointestinal involvement causing bleeding and/ or obstruction, ${ }^{4}$ and genitourinary and/or gynecologic involvement causing bleeding. ${ }^{5,6}$

PRT accounts for between $30 \%$ and $50 \%$ of courses of radiotherapy delivered. ${ }^{3}$ These courses of $\mathrm{RT}$ typically require urgent evaluation since patients are seen because of new and/or progressive symptoms that give cause for concern. The urgency of presentation requires radiation oncologists and the departments receiving these patients to be equipped to manage these cases efficiently and effectively.
Furthermore, the types of cases seen, including PRT indications and related symptoms requiring management, inform the training of radiation oncology physicians as well as nursing and other clinical staff. Finally, characterizing the types of urgent PRT cases that are seen can also guide research and quality improvement endeavors for advanced cancer care in radiation oncology settings.

There is currently a paucity of data characterizing the types and frequencies of urgent PRT indications in patients who present to radiation oncology departments, as well as a lack of data detailing the related symptoms radiation oncology clinicians are managing. The aim of this study was to characterize the types and frequencies of urgent PRT consultations and the related symptoms that radiation oncologists are managing as part of patient care.

Accepted for publication July 17, 2018. Correspondence: Muhammed M Fareed, MD; mmfareed@bwh.harvard.edu. Disclosures: The authors report no disclosures or conflicts of interest. JCSO 2018;16(4):e193-e199. (02018 Frontline Medical Communications. doi: https://doi.org/10.12788/jcso.0415 


\section{Methods}

Based on national palliative care practice and national oncology care practice guidelines, ${ }^{7,8}$ we designed a survey to categorize the cancer-related palliative care issues seen by radiation oncologists. Physical symptoms, psychosocial issues, cultural consideration, spiritual needs, care coordination, advanced-care planning, goals of care, and ethical and legal issues comprised the 8 palliative care domains that we evaluated. A survey was developed and critically evaluated by 3 investigators (MK, VL, TB). Each palliative care domain was ranked by clinicians by its relevance (5-point Likert scale [range, 1-5]: 1, Not Relevant, to 5, Extremely Relevant) to the patient's care point in radiation oncology. In addition, 31 palliative care subissues related to the primary domains were identified by clinicians based on their presence (Yes, No, Not Assessed). Clinicians were also asked whether the consulted patient's metastatic cancer diagnosis was established (longer than 1 month) or new (within the last 1 month). In addition, clinicians noted whether the patient was returning to active oncologic care (eg, chemotherapy) or to no further anticancer therapies (eg, hospice care) after RT consultation and intervention (if deemed necessary).

The survey's face and content validity, ease of completion, and time of completion was assessed by a panel of 7 clinicians with expertise in medical oncology, radiation oncology, palliative care, and/or survey construction. The survey was then sent in a sequential manner to 1 member of the panel at a time after incorporating each panel member's initial comments. After each panel member's review, the survey was edited until 2 consecutive panel members had no further suggestions for improvement.

After receiving approval from the institutional review boards of participating radiation oncology centers, we electronically surveyed radiation oncology clinicians who were conducting PRT consultations. From May 19 to September 26, 2014, all consultations were evaluated prospectively for consideration of PRT performed by a dedicated PRT service at 3 centers (a large academic cancer center and 2 participating clinicians at affiliated regional hospitals). The consultations for patients aged 18 years or older with incurable, metastatic cancers were considered eligible. The consulting clinician was e-mailed a survey for completion within 5 business days immediately after each PRT consult. Three reminders to complete the survey were sent during the 5-businessday interval. Over the entire study period, 162 consecutive patients were identified, resulting in 162 surveys being sent to 15 radiation oncology clinicians, including nurse practitioners, resident physicians, and attending physicians. Each clinician received a $\$ 25$ gift card for participating, regardless of the number of surveys completed. In total, 140 of the 162 surveys were returned, resulting in a response rate of $86 \%$.
TABLE Patient characteristics and case information ( $N=140$ )

Characteristic/information

n (\%)

\begin{tabular}{|c|c|}
\hline \multicolumn{2}{|l|}{ Gender } \\
\hline Men & $79(56)$ \\
\hline Women & $61(44)$ \\
\hline \multicolumn{2}{|l|}{ Age, y } \\
\hline$\leq 50$ & $29(20)$ \\
\hline $50-65$ & $56(41)$ \\
\hline$\geq 65$ & 55 (39) \\
\hline \multicolumn{2}{|l|}{ Marital Status } \\
\hline Married & $86(62)$ \\
\hline Single/widowed/divorced & $54(38)$ \\
\hline \multicolumn{2}{|l|}{ Race } \\
\hline White & $99(70)$ \\
\hline Other & $41(30)$ \\
\hline \multicolumn{2}{|l|}{ Cancer type } \\
\hline Lung & $39(28)$ \\
\hline Prostate & $14(10)$ \\
\hline Breast & $18(13)$ \\
\hline Melanoma & $14(10)$ \\
\hline Other & 55 (39) \\
\hline \multicolumn{2}{|l|}{ ECOG Performance Status ${ }^{a}$} \\
\hline $0-1$ & $77(57)$ \\
\hline 2 & $39(29)$ \\
\hline 3 & $14(10)$ \\
\hline 4 & $4(3)$ \\
\hline \multicolumn{2}{|l|}{$\begin{array}{l}\text { Clinician performing patient } \\
\text { initial consult evaluation }\end{array}$} \\
\hline Nurse practitioner ${ }^{b}$ & $72(51)$ \\
\hline Resident physician & $51(36)$ \\
\hline Attending physician & $17(12)$ \\
\hline \multicolumn{2}{|l|}{$\begin{array}{l}\text { Practice type location } \\
\text { of patient consultations }\end{array}$} \\
\hline Academic & $127(91)$ \\
\hline Hybridc & $13(9)$ \\
\hline
\end{tabular}

ECOG, Eastern Cooperative Oncology Group

${ }^{\circ} 0$, fully active; 1 , restricted in physically strenuous activity but ambulatory and able to carry out light/sedentary work; 2 , ambulatory and capable of self-care but unable to carry out any work activities; 3 , capable of only limited self-care and up and about more than $50 \%$ of waking hours; confined to bed or chair more than $50 \%$ of waking hours; 4 , completely disabled, cannot carry out any self-care, and totally confined to bed or chair. 'N Nurse practitioner and residents did the initial patient intake, followed by an attending MD seeing the patient. 'Community practice affiliated with an academic center.

The investigators then collected patient demographics (age, gender, race, marital status) and disease characteristics (primary cancer type, Eastern Cooperative Oncology 
Group Performance Status, reasons for urgent RT consult, physical symptoms requiring management at presentation, patient's place in illness trajectory, and RT recommendation) pertaining to each completed survey from the electronic medical record. Urgent consultations were defined as any patients who needed to be seen on the same day or within a few days of the consult request.

The descriptive statistics of all these data were calculated in terms of frequencies and percentage of categorical variables. Chi-squared statistics, Fisher exact test, and nonparametric rank sum tests were applied to various categories to determine any statistically significant differences between groups.

\section{Results}

In total, 162 patients were seen in consultation for PRT during the 19-week enrollment period, or an average of 8.7 consults a week. Of that total, surveys for 140 patients were returned (Table). The median patient age was 63 years (range, 29-89 years). A sizeable minority (20\%) was 50 years or younger. The most common cancer diagnosis was lung cancer $(28 \%)$, followed by breast $(13 \%)$ and prostate (10\%) cancers, melanoma (10\%), and sarcoma (7\%). Other diagnoses accounted for the remaining $32 \%$.

\section{Timing of PRT consult in cancer trajectory}

The points in the advanced cancer illness trajectory at which patients were seen for PRT evaluation are shown in Figure 1. Most patients (63\%) were seen for a PRT evaluation at the time of an established diagnosis $(>1$ month after diagnosis of metastatic cancer) and were continuing to further cancer therapies. An additional 19\% of patients with an established diagnosis proceeded to hospice or end-oflife care after the PRT evaluation. A notable minority of patients $(18 \%)$ were seen for a PRT evaluation at the time of a new diagnosis $(<1$ month of diagnosis of metastatic cancer), and of those, $17 \%$ went on to receive anticancer therapy after the PRT evaluation and $1 \%$ proceeded to hospice or end-of-life care.

\section{Characteristics of PRT consults and symptoms at presentation}

The primary reasons for urgent consultation for PRT are shown in Figure 2. Cancerrelated pain (57\%), brain metastases (29\%), and malignant spinal cord or cauda equina compression (13\%) were the predominant reasons for consults. Notable minorities were seen for tumor-related dyspnea (10\%), bleeding (8\%), and bone fractures (4\%). Symptoms requiring management as part of the radiation oncology consultation are shown in Figure 3. The most commonly managed symptoms were pain (69\%), neurologic symptoms (51\%), and fatigue (49\%). Management of gastrointestinal symptoms was the next most common category (39\%), with constipation being the most common gastrointestinal symptom (21\%), followed by nausea and/or vomiting (12\%) and dysphagia (6\%).

\section{PRT recommendations and targets}

Recommendations regarding PRT are shown in Figure 4A. Of the total 140 patients, $18(13 \%)$ were not recommended for RT. Of the 122 patients for whom PRT was recommended, 11 (9\%) received RT at more than 1 site. Figure 4B shows sites of PRT. Of the 133 sites treated in 122 patients, 61 (46\%) were bone, 39 (29\%) were brain, 11 (8\%) were lung, and $22(17 \%)$ were other soft-tissue sites.

\section{Discussion and conclusions}

The present study provides a descriptive overview of urgent metastatic cancer patient presentations to radiation oncology clinicians through a comprehensive evaluation of 140 consults for PRT. The most common reasons for urgent evaluation were cancer-related pain (57\%), but brain metastases (29\%), spinal cord compression (13\%), and respiratory symptoms (10\%) were also common. Other less-common indications included cancer-related dysphagia, bleeding, and poststabilization management of bone fractures. The most common symptoms requiring management by radiation oncology clinicians were pain (69\%), neurologic symptoms (51\%), and fatigue (49\%). The study also provides a comprehensive characteriza-

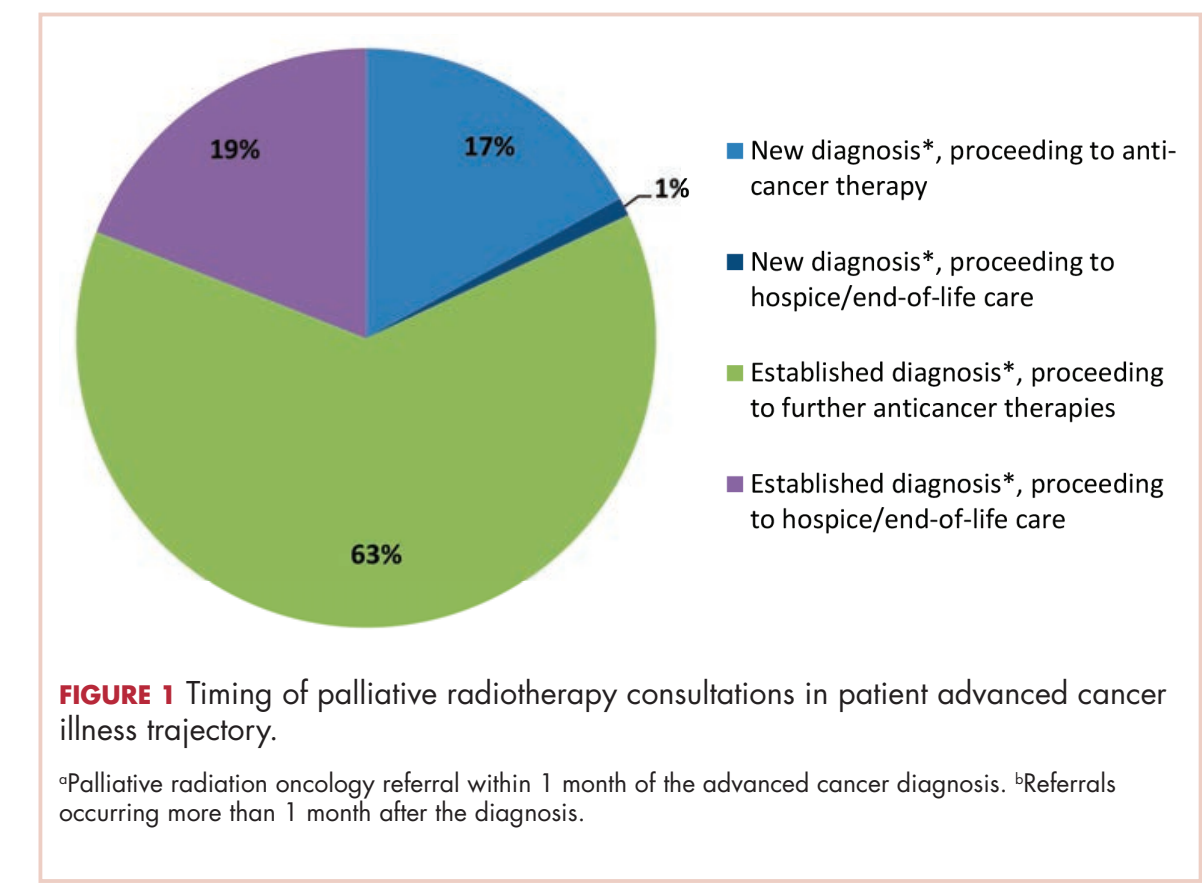



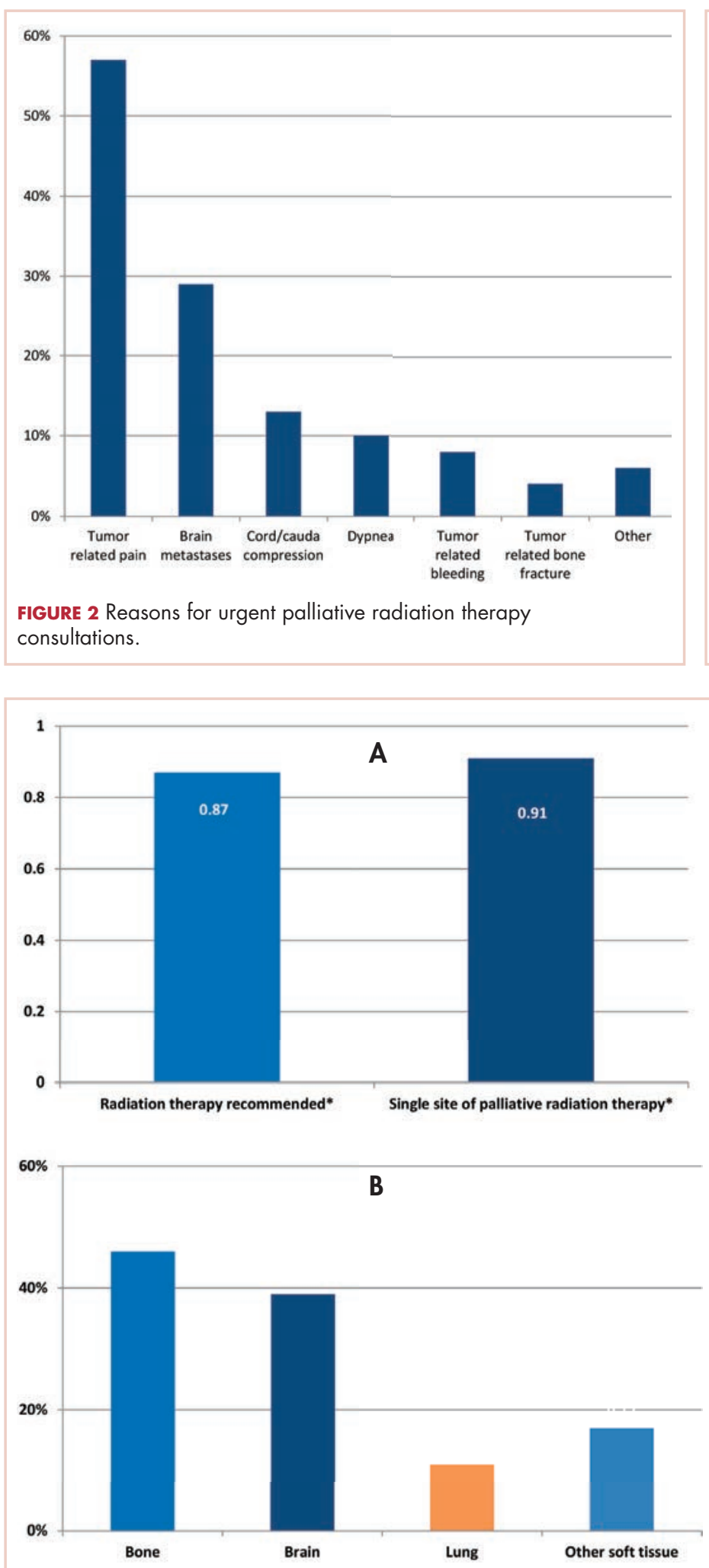

FIGURE 4 A, Palliative radiation therapy recommendations. B, Sites of palliative radiation therapy delivery.

${ }^{\circ}$ Not recommended in $13 \%$ of patients. ${ }^{6}$ To multiple sites in $9 \%$ of patients.

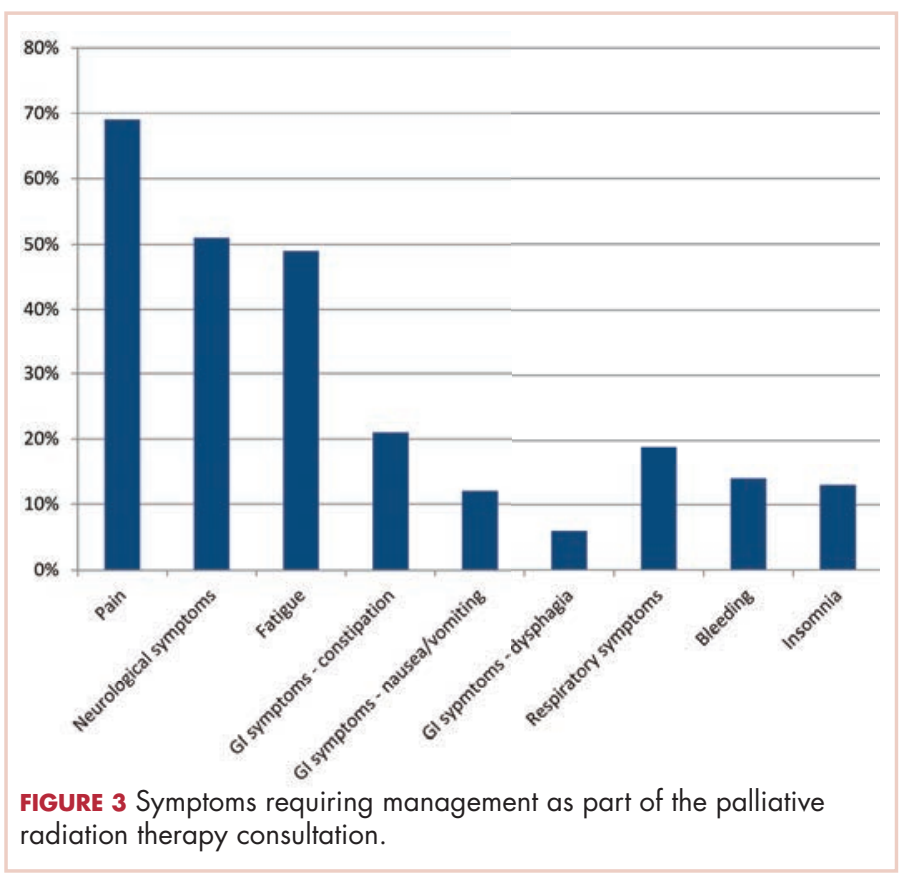

tion of the timeframe of PRT consultation and the treatment recommendations in this cohort. Though most PRT consults occurred at the time of an established metastatic cancer diagnosis and before further anticancer therapies, sizeable minorities occurred at the time of a new diagnosis of metastatic cancer (18\%) and before comfortfocused, end-of-life care and no further anticancer therapies (20\%). Most patients (87\%) were recommended PRT, and of those recommended RT, $11 \%$ received RT to more than 1 site. The most common PRT sites were to bone (46\%), followed by brain (29\%), nonlung soft-tissue sites (17\%), and lung (8\%). This comprehensive description of the day-to-day urgent, advanced cancer care issues seen and managed in radiation oncology practice can help guide PRT clinical structures, education, research, and quality improvement measures in clinical practice.

Our study provides an insight into urgent symptoms encountered by radiation oncology practitioners during their routine practice. Cancer-related pain remains the most common symptom requiring management. Given the frequency with which pain management is needed among PRT patients, this study highlights the need for radiation oncologists to be well trained in symptom management, particularly as the pain response to RT can often take several days. However, studies suggest that cancer-related pain is not frequently managed by radiation oncologists. ${ }^{9}$ For example, findings from an Italian study showed that the involvement of radiation oncologists in cancer pain management remains minimal compared with other medical professionals; during the treatment course, only half of the radiation oncologists implemented specific treatment for breakthrough 
pain. ${ }^{10} \mathrm{~A}$ nationwide survey in the United States implicated a number of barriers to adequate pain management, including poor assessment by the physician, reluctance in prescribing opioid analgesics, perceived excessive regulation, and patient reluctance to report pain. ${ }^{11}$ Notably, in a survey of the Radiation Therapy Oncology Group study physicians, $83 \%$ believed cancer patients with pain were undermedicated, and $40 \%$ reported that pain relief in their own practice setting was suboptimal. Furthermore, in the treatment plan, adjuvants and prophylactic side effect management were frequently not used properly. ${ }^{12}$ Education of radiation oncologists in pain assessment and management is key to overcome these barriers and to ensure adequate pain management and quality of life for patients in radiation oncology.

The next most common reason for which patients presented for palliative radiation oncology consultation was for central nervous system (CNS) metastatic disease, including brain metastases and spinal cord compression. Correspondingly, the next most common issue requiring management was neurologic symptoms. Management of CNS disease is becoming increasingly complex, and it benefits from multidisciplinary evaluation to guide optimal and personalized care for each patient, including medical oncology, radiation oncology, neurosurgery and/or orthopedic spine surgery, and palliative medicine. Treatment options include supportive care or corticosteroids alone, surgical resection, whole-brain RT, and/or radiosurgery or stereotactic RT alone. These treatment options are considered on the basis of global patient factors, such as prognosis, together with metastatic-site-specific factors, such as site-related symptoms and the number of metastatic diseases or the burden of the disease. ${ }^{13}$ For example, the use of the diagnosis-specific Graded Prognostic Assessment index to predict life expectancy can help tailor management of brain metastases based on performance status, age, number of brain metastases, extracranial metastases, and cancer type. Highlighting the complexity of this common PRT presentation, Tsao and colleagues showed that there was a lack of uniform agreement among radiation oncologists for common management issues in patients with brain metastatic disease. ${ }^{14}$

For metastatic spinal cord or cauda equina compression and the associated neurologic symptoms, initiation of immediate corticosteroids and implementation of local therapy within 24 hours of presentation is paramount, ${ }^{15}$ highlighting the need for rapid, comprehensive care decision-making for these patients. Treatment options that must be weighed include the potential benefit of upfront decompressive surgery, as supported by a randomized controlled trial by Patchell and colleagues ${ }^{16}$ for patients who are surgical candidates with true cord or cauda compression and have at least 1 neurologic symptom, a prognosis of $\geq 3$ months, paraplegia of no longer than 48 hours, and no previous RT to the site or brain metastases. Compared with the RT alone, patients receiving surgery before RT had improved ambulatory status and overall survival. Hence, neurosurgical or orthopedic consultation should be standard in the evaluation of metastatic spinal cord or cauda equina compression patients. However, patients frequently do not meet these criteria, and corticosteroids and RT alone are considered. In addition to playing a role in surgical decision-making, prognosis also has a key role in decision-making about the RT fractionation. Short-course RT $(8 \mathrm{~Gy} \times 1)$ is as effectual as longer-course regimens $(3 \mathrm{~Gy} \times 10)$ in terms of motor function. ${ }^{17,18}$ However, more dose-intense or longer-course regimens, such as $3 \mathrm{~Gy} \times 10$, have been shown to have more durability beyond about 6 months and are therefore considered for intermediate to good prognosis. ${ }^{18}$ The common urgent presentation of CNS metastatic disease to radiation oncology clinics together with the complexity of management and urgency of care decision-making point to the need for dedicated structures of care for these patients in radiation oncology settings. For example, dedicated PRT programs, such as the Rapid Response Radiotherapy Program in Toronto and the Supportive and Palliative Radiation Oncology service in Boston, have demonstrated improved quality of care for patients being urgently evaluated for PRT. ${ }^{19}$

Following management of pain and neurologic symptoms, clinicians were faced with managing fatigue in nearly half of the patients (49\%). The prevalence of fatigue among cancer patients and its impact on quality of life ${ }^{20}$ highlight the need for this key symptom to be addressed throughout the continuum of cancer care. National Comprehensive Cancer Network guidelines provide a comprehensive framework for addressing cancer-related fatigue. ${ }^{7}$ However, cancer-related fatigue is a largely underreported, underestimated, and thus undertreated problem. ${ }^{20}$ In a nationwide survey of members of the American Society for Radiation Oncology, radiation oncologists reported being significantly less confident in managing fatigue compared with managing other common symptoms. ${ }^{21}$ Furthermore, in a national survey of radiation oncology trainees, $67 \%$ of respondents indicated that they were not at all minimally or somewhat confident in their ability to manage fatigue symptoms. The frequency of this symptom together with the demonstrated need for improved education in fatigue management point to a need for radiation oncology palliative educational structures to include dedicated emphasis on managing fatigue in addition to other commonly encountered symptoms, such as pain.

Patients evaluated for PRT are seen across the trajectory of their metastatic cancer diagnosis. In our study, patients presented at all stages in their advanced cancers. These include patients seen at the time of initial diagnosis of can- 
cer as well as those seen near the end of life when endof-life care planning was underway. The broad spectrum of timing of PRT care underscores that radiation oncologists must be prepared to handle generalist palliative care issues encountered throughout the trajectory of advanced cancer care and hence need comprehensive education in generalist palliative care competencies. These include symptom management, end-of-life care coordination, and communication or goals-of-care discussions. Notably, a recent national survey of radiation oncology residents indicated that most residents, $77 \%$ on average, perceived their educational training as suboptimal across domains of generalist palliative care competencies needed in oncology practice. ${ }^{22}$ Furthermore, a majority (81\%) desired greater palliative care education within training.

The most common sites treated in this study were bone, brain, and lung sites. These data provide guidance to both education and research initiatives aiming to advance PRT curriculum and care structures within departments. For example, a same-day simulation and radiation treatment program developed at Princess Margaret Hospital Palliative Radiation Oncology Program (Ontario, Canada) aids in providing streamlined care for patients with bone metastases, the most common presentation for PRT. ${ }^{23}$ Furthermore, education and research in the application of PRT techniques to bone, brain, and thoracic disease cover the majority of PRT presentations. It is notable, however, that $17 \%$ were other soft tissue body sites.

\section{References}

1. Chow E, Harris K, Fan G, Tsao M, Sze WM. Palliative radiotherapy trials for bone metastases: a systematic review. J Clin Oncol. 2007;25(11):1423-1436.

2. van Oorschot B, Rades D, Schulze W, Beckmann G, Feyer P. Palliative radiotherapy--new approaches. Semin Oncol. 2011;38(3):443-449.

3. Simone CB II, Jones JA. Palliative care for patients with locally advanced and metastatic non-small cell lung cancer. Ann Palliat Med. 2013;2(4):178-188.

4. Cihoric N, Crowe S, Eychmüller S, Aebersold DM, Ghadjar P. Clinically significant bleeding in incurable cancer patients: effectiveness of hemostatic radiotherapy. Radiat Oncol. 2012;7:132.

5. Duchesne GM, Bolger JJ, Griffiths GO, et al. A randomized trial of hypofractionated schedules of palliative radiotherapy in the management of bladder carcinoma: results of medical research council trial BA09. Int J Radiat Oncol Biol Phys. 2000;47(2):379-388.

6. Onsrud M, Hagen B, Strickert T. 10-Gy single-fraction pelvic irradiation for palliation and life prolongation in patients with cancer of the cervix and corpus uteri. Gynecol Oncol. 2001;82(1):167-171.

7. NCCN Guidelines(R) Updates. J Natl Compr Canc Netw. 2013;11(9):xxxii-xxxvi.

8. Colby WH, Dahlin C, Lantos J, Carney J, Christopher M. The National Consensus Project for Quality Palliative Care Clinical Practice Guidelines Domain 8: ethical and legal aspects of care. HEC Forum. 2010;22(2):117-131.

9. Stockler MR, Wilcken NR. Why is management of cancer pain still a problem? J Clin Oncol. 2012;30(16):1907-1908.

10. Caravatta L, Ramella S, Melano A, et al. Breakthrough pain management in patients undergoing radiotherapy: a national survey on behalf of the Palliative and Supportive Care Study Group. Tumori. 2015;101(6):603-608.

\section{Limitations}

There are a few limitations to this study. First, this is a survey-based study conducted at a single academic center within an urban setting and surrounding community regions, which affects its generalizability. Second, this study presents perspectives of radiation oncology practitioners evaluating patients and does not directly reflect patient perceptions or report of symptoms. Third, the data provided by this study are solely descriptive in nature. However, this can guide hypothesis-driven research regarding the evaluation and management of urgent palliative care issues encountered by radiation oncology clinicians and suggest educational objectives to address the needs of these patients.

\section{Conclusions}

Radiation oncologists are involved throughout the trajectory of care for advanced cancer patients. Furthermore, they manage a variety of urgent oncologic issues, most commonly metastases causing pain, brain metastases, and spinal cord or cauda equina compression. Radiation oncologists also manage many cancer-related symptoms, mostly pain, neurologic symptoms, fatigue, and gastrointestinal symptoms. These findings point toward the need for palliative care to be well integrated into radiation oncology training curricula and the need for dedicated care structures that enable rapid and multidisciplinary palliative oncology care within radiation oncology departments.

11. Breuer B, Fleishman SB, Cruciani RA, Portenoy RK. Medical oncologists' attitudes and practice in cancer pain management: a national survey. J Clin Oncol. 2011;29(36):4769-4775.

12. Cleeland CS, Janjan NA, Scott CB, Seiferheld WF, Curran WJ. Cancer pain management by radiotherapists: a survey of radiation therapy oncology group physicians. Int J Radiat Oncol Biol Phys. 2000;47(1):203-208.

13. Tsao MN, Rades D, Wirth A, et al. Radiotherapeutic and surgical management for newly diagnosed brain metastasis(es): an American Society for Radiation Oncology evidence-based guideline. Pract Radiat Oncol. 2012;2(3):210-225.

14. Tsao MN, Rades D, Wirth A, et al. International practice survey on the management of brain metastases: third international consensus workshop on palliative radiotherapy and symptom control. Clin Oncol (R Coll Radiol). 2012;24(6):e81-e92.

15. Tang V, Harvey D, Park Dorsay J, Jiang S, Rathbone MP. Prognostic indicators in metastatic spinal cord compression: using functional independence measure and Tokuhashi scale to optimize rehabilitation planning. Spinal Cord. 2007;45(10):671-677.

16. Patchell RA, Tibbs PA, Regine WF, et al. Direct decompressive surgical resection in the treatment of spinal cord compression caused by metastatic cancer: a randomised trial. Lancet. 2005;366(9486):643-648.

17. Rades D, Stalpers LJ, Veninga T, et al. Evaluation of five radiation schedules and prognostic factors for metastatic spinal cord compression. J Clin Oncol. 2005;23(15):3366-3375.

18. Rades D, Stalpers LJ, Hulshof MC, et al. Comparison of $1 \times 8$ Gy and $10 \times 3 \mathrm{~Gy}$ for functional outcome in patients with metastatic spinal cord compression. Int J Radiat Oncol Biol Phys. 2005;62(2):514-518.

19. Dennis K, Linden K, Balboni T, Chow E. Rapid access palliative 
radiation therapy programs: an efficient model of care. Future Oncol. 2015;11(17):2417-2426.

20. Kapoor A, Singhal MK, Bagri PK, Narayan S, Beniwal S, Kumar HS. Cancer related fatigue: a ubiquitous problem yet so under reported, under recognized and under treated. South Asian J Cancer. 2015;4(1):21-23.

21. Wei RL, Mattes MD, Yu J, et al. Attitudes of radiation oncologists toward palliative and supportive care in the United States: report on national membership survey by the American Society for Radiation Oncology (ASTRO). Pract Radiat Oncol. 2017;7(2):113-119.

22. Krishnan M, Racsa M, Jones J, et al. Radiation oncology resident palliative education. Pract Radiat Oncol. 2017;7(6):e439-e448.

23. McDonald R, Chow E, Lam H, Rowbottom L, Soliman H. International patterns of practice in radiotherapy for bone metastases: a review of the literature. J Bone Oncol. 2014;3(3-4):96-102. 